\title{
Hyperactivity Scale
}

National Cancer Institute

\section{Source}

National Cancer Institute. Hyperactivity Scale. NCI Thesaurus. Code C121282.

A rating scale included in the Behavior Assessment System for Children that measures

the tendency to be overly active, rush through work or activities, and act without thinking. 